\title{
Human CD72 splicing isoform responsible for resistance to systemic lupus erythematosus regulates serum immunoglobulin level and is localized in endoplasmic reticulum
}

\author{
Yuki Hitomi ${ }^{1,2,3}$, Takahiro Adachi ${ }^{1,2}$, Naoyuki Tsuchiya ${ }^{5}$, Zen-Ichiro Honda ${ }^{4}$, Katsushi Tokunaga ${ }^{3}$
} and Takeshi Tsubata ${ }^{1,2,6^{*}}$

\begin{abstract}
Background: CD72 is an inhibitory co-receptor expressed on B cells. We previously demonstrated significant association of the polymorphism of the CD72 gene with susceptibility to human systemic lupus erythematosus (SLE) in individuals carrying a SLE-susceptible FCGR2B genotype (FCGR2B-232Thr/Thr). The human CD72 locus generates a splicing isoform that lacks exon 8 (CD72 $\Delta$ ex8) as well as full-length CD72 (CD72fl), and the CD72 polymorphism regulates exon 8 skipping.

Results: Here we demonstrated that individuals carrying the disease-protective CD72 genotype exhibit significantly lower serum immunoglobulin levels than do individuals carrying other CD72 genotypes $(P<0.05)$. Although expression level of CD72fl in the peripheral blood B cells was similar regardless of CD72 genotype, the protein level of CD72 2 ex8 was increased in individuals carrying the disease-protective CD72 genotype, suggesting a crucial role of CD72 2 ex8 in regulation of antibody production. By expressing these human CD72 isoforms in mouse cell lines, we further demonstrated that CD72 $\triangle$ ex8 is accumulated in endoplasmic reticulum (ER) and fails to regulate $B C R$ signaling whereas human CD72fl is efficiently transported to the cell surface and inhibits signaling through the B cell antigen receptor (BCR), as is the case for mouse CD72.
\end{abstract}

Conclusion: Human CD72 polymorphism appears to regulate antibody production as well as susceptibility to SLE by regulating expression of ER-localizing CD72 $\Delta$ ex8.

Keyword: Polymorphism, Exon skipping, C-type lectin domain

\section{Background}

CD72, a $45 \mathrm{kDa}$ type II membrane protein expressed on $\mathrm{B}$ cells, is an inhibitory co-receptor that regulates signaling through the $\mathrm{B}$ cell antigen receptor (BCR) [1-6]. Both human and mouse CD72 contains a C-type lectinlike domain in the extracellular region and an immunoreceptor tyrosine-based inhibition motif (ITIM) in the cytoplamic region [1-3]. Mouse CD72 negatively regulates $\mathrm{BCR}$ signaling by recruiting Src homology 2

\footnotetext{
* Correspondence: tsubata.imm@mri.tmd.ac.jp

'Laboratory of Immunology, School of Biomedical Science, Tokyo Medical and Dental University, 1-5-45 Yushima, Bunkyo-ku, Tokyo 113-8510, Japan ${ }^{2}$ Department of Immunology, Medical Research Institute, Tokyo Medical and Dental University, 1-5-45, Yushima, Bunkyo-ku, Tokyo 113-8510, Japan Full list of author information is available at the end of the article
}

domain-containing protein tyrosine phosphatase-1 (SHP-1) at ITIM [4-6]. However, the signaling function of human CD72 remains unknown.

Four human CD72 polymorphisms have been identified in the upstream regulatory region and introns [7]. These $C D 72$ polymorphisms constitute two major haplotypes, $C D 72 * 1$ and $C D 72 * 2$. We previously demonstrated that FCGR2B-Ile232Thr, a gene polymorphism of Fc $\gamma$ RIIb, is significantly associated with SLE in Asian populations, and is a risk factor for SLE only in individuals with $C D 72 * 1 / 1[7,8]$. This finding indicates that $C D 72 * 2$ confers resistance to SLE in individuals carrying FCGR2B-Ile232Thr. Two polymorphisms in intron 8 regulate generation of an alternative splicing isoform

\section{Biomed Central}


(CD72 2 ex8) that skips exon 8 independently; probably act in combination as cis-acting intronic splicing enhancer (ISE) or silencer (ISS) [7]. Exon 8 encodes the Cterminal part of the C-type lectin-like domain and the stop codon, and skipping of it results in replacement of the $\mathrm{C}$-terminal part of the $\mathrm{C}$-type lectin-like domain by a sequence encoded in exon 9 in CD72 $\Delta$ ex8. The ratio of mRNA level of CD72 $\triangle \mathrm{ex} 8$ to that of full-length CD72 $(\mathrm{CD} 72 \mathrm{fl})$ is strikingly higher in B cells from individuals with the $C D 72 * 2 / 2$ or $C D 72 * 1 / 2$ genotype than in those with $C D 72 * 1 / 1$ [7]. Because there are no substitutions in exons among different $C D 72$ haplotypes, these findings strongly suggest that increased CD72 $\Delta$ ex8 level, decreased CD72fl level, or both are responsible for the resistance of $C D 72 * 2$-carrying individuals to SLE.

In the present study, we addressed the functional properties of CD72 isoforms. Analysis of healthy individuals revealed that those with $C D 72 \% 1$ express a significantly lower level of the CD72 $\Delta$ ex 8 protein in B cells, and show the higher level of serum immunoglobulins than those carrying $C D 72 * 2$, suggesting that $C D 72 \Delta \operatorname{ex} 8$ regulates the immunoglobulin level as well susceptibility to SLE. Analysis using B cell transfectants expressing CD72 $\Delta$ ex 8 demonstrated that CD72 $\Delta$ ex 8 does not regulate $\mathrm{BCR}$ signaling but accumulates in the endoplasmic reticulum (ER). Thus, the CD72 polymorphism regulates antibody production and autoimmunity by modulating the level of ER-localizing CD72 $\Delta \mathrm{ex} 8$.

\section{Methods}

\section{Plasmids}

cDNAs including the entire coding region of human CD72fl or CD72 $\Delta$ ex8 but not nucleotides for the stop codon were obtained by RT-PCR from peripheral blood mononuclear cells (PBMCs) with a pair of specific primers (5'-GCA GAG CTG CTC AGG ACC AT-3' and $5^{\prime}$-ACC CCA TTC TAC CAT GGG AA-3'). The cDNAs encoding $\mathrm{CD} 72 \mathrm{fl}$ and $\mathrm{CD} 72 \Delta \mathrm{ex} 8$ were inserted with a pair of oligonucleotides encoding FLAG-tag into the retrovirus expression vector pMX-ires-GFP, and the resulting plasmids were termed pMX-CD72fl and pMXCD72 $\Delta$ ex8, respectively.

The retrovirus expression plasmids pMX-CD72flYF and pMX-CD72 $\triangle$ ex8YF encoding the mutants of CD72fl and $C D 72 \Delta$ ex 8 , in which tyrosine ${ }^{7}$ is replaced by phenylalanine, were generated by PCR-based site-directed mutagenesis using a specific primer set $\left(5^{\prime}-\right.$ GCA GAT CTG AGG TTT GTG AA $-3^{\prime}$ and $5^{\prime}$ - AAA GGT GAT GGC CTC AGC CA - $3^{\prime}$ ).

\section{Cells}

The mouse B cell lines WEHI-231 and K46 $\mathrm{Nv}$ and the human B cell line Raji were described previously $[9,10]$, and cultured in RPMI 1640 medium supplemented with
$10 \%$ FCS, $50 \mu \mathrm{M}$ 2-ME, and $1 \mathrm{mM}$ glutamine. The mouse fibroblast cell line Balb/c-3T3 was cultured in DMEM medium supplemented with $10 \%$ FCS and $1 \mathrm{mM}$ glutamine. Retrovirus-mediated gene transfer was performed as described previously [9].

PBMCs were obtained from unrelated healthy Japanese living in the central part of Japan where genetic background is shown to be relatively homogeneous [11]. Informed consents were obtained from these indiciduals prior to collecting samples. Peripheral B lymphocytes were isolated from PBMCs by an autoMACS cell sorter (Miltenyi Biotec, Auburn, CA) using the B cell isolation kit II. This study was approved by the Research Ethics Committees of the Graduate School of Medicine, The University of Tokyo.

\section{Genotyping}

Human CD72 haplotype was determined by genotyping CD72-VNTR, the tag polymorphism, by PCR-SSLP method as described previously [7]. FCGR2B-Ile232Thr was genotyped by nested PCR and fluorescence resonance energy transfer (FRET) technology as described previously [12].

\section{Serum IgG level}

Serum IgG levels in healthy individuals were measured by turbidimetric immunoassay.

\section{Flow cytometry}

Peripheral blood B cells were incubated with FITC-labeled anti-human CD72 mAb J4-117 (BD Biosciences, San Jose, CA). B cell transfectants were incubated with rabbit antiFLAG Ab (Cell Signaling Technology, Danvers, MA), followed by reaction with PE-labeled goat anti-rabbit IgG (Southern Biotech, Birmingham, AL). Alternatively, transfectants were stained with NP-conjugated PE. Cells were then analyzed by flow cytometry using a FACSCalibur (BD Biosciences).

\section{Generation of anti-CD72 $\Delta$ ex8 antibody}

Rabbits were immunized with the peptide specific for CD72 $\Delta$ ex8 (Ala-Asp-Pro-His-Leu-Thr-Leu), and serum IgG was purified from rabbit serum by ammonium sulfate precipitation. The specificity of the antibody was confirmed by Western blotting of total cell lysates of COS-7 CD72 2 ex 8 transfectant using pre-immune and immune serum.

\section{Immunoprecipitation and Western blotting}

Cells were stimulated with either $0.2 \mu \mathrm{g} / \mathrm{ml} \mathrm{NP}_{15}$-coupled BSA (NP-BSA) or $10 \mu \mathrm{g} / \mathrm{ml}$ anti-IgM Ab, and were lysed in Triton X-100 lysis buffer $[9,10]$. Lysates were immunoprecipitated with anti-FLAG Ab (Sigma-Aldrich, St. Louis, $\mathrm{MO}$ ) together with protein G-Sepharose (GE Healthcare 
Life Sciences, Piscataway, NJ). Total cell lysates or immunoprecipitates were separated on SDS-PAGE and were transferred to polyvinylidene difluoride membranes. Membranes were incubated with anti-phosphotyrosine $\mathrm{mAb}$ 4G10 (Millipore), anti- $\beta$-tubulin mAb TUB2.1 (Seikagaku Kogyo, Tokyo, Japan), rabbit anti-FLAG Ab, rabbit antiCD72 Ab (Santa Cruz Biotechnology, Santa Cruz, CA), rabbit anti-phospho ERK Ab (Cell Signaling Technology), rabbit anti-SHP-1 Ab (Santa Cruz Biotechnology), or rabbit anti-CD72 $\triangle \mathrm{ex} 8 \mathrm{Ab}$. Proteins were visualized with an ECL system.

\section{Measurement of intracellular calcium mobilization}

Cells were incubated in culture medium containing $5 \mu \mathrm{g} / \mathrm{ml}$ fluo-4-AM (Molecular Probes) for $30 \mathrm{~min}$. Cells were stimulated with $0.2 \mu \mathrm{g} / \mathrm{ml} \mathrm{NP-BSA}$, and were analyzed by flow cytometry using a FACSCalibur.

\section{Assay of apoptotic cells}

Cells were incubated with thapsigargin or staurosporine for $24 \mathrm{~h}$ and $48 \mathrm{~h}$, and cells containing hypodiploid DNA were measured by flow cytometry [10].

\section{Immunocytochemistry}

Cells were fixed in 4\% PFA for 15 min, washed with PBS, and permeabilized with $0.1 \%$ Triton X-100 for $15 \mathrm{~min}$. Cells were then washed with PBS, blocked with 2\% BSA for $30 \mathrm{~min}$, and stained for FLAG-tagged human CD72 and organelle-specific marker proteins in ER, mitochondria, Golgi apparatus, early endosomes, and late endosomes using rabbit anti-FLAG $\mathrm{Ab}$, antiKDEL mAb 10C3 (Stressgen, MI), anti-cytochrome C mAb 6H2.B4 (BD Biosciences), anti-GM130 mAb 35 (BD Biosciences), anti-transferrin receptor mAb H68.4 (Invitrogen-Life Technologies), and anti-lamp1 mAb 1D4B (Southern Biotech), respectively. Cells were then analyzed by laser scanning confocal microscopy (Model LSM 510; Carl Zeiss Inc., Jena, Germany).

\section{Statistical analysis}

The serum IgG level among CD72 genotypes was analyzed using regression analysis and Kruskal-Wallis rank sum test. Relative expression of $C D 72 \Delta$ ex8 protein product between $C D 72$ genotype was analyzed using MannWhitney U test. Otherwise, Student's t-test was used for statistical analysis.

\section{Results}

Decreased serum immunoglobulin levels in CD72*2-carrying individuals

To address whether the $C D 72$ polymorphism regulates humoral immunity, we measured the serum IgG levels in 30 healthy volunteers. We genotyped CD72 IVS8VNTR which was the tag polymorphism for human

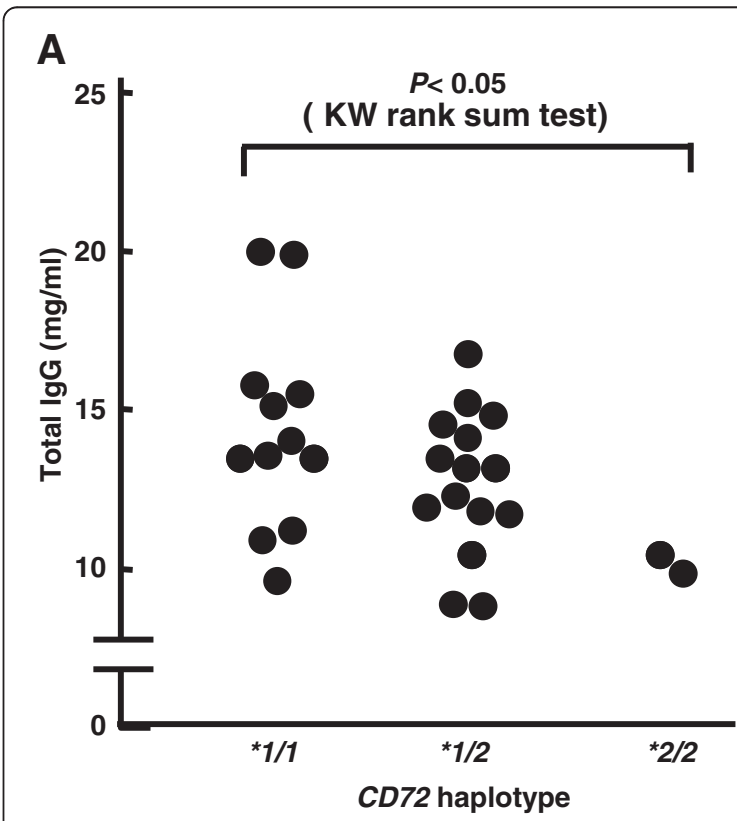

B

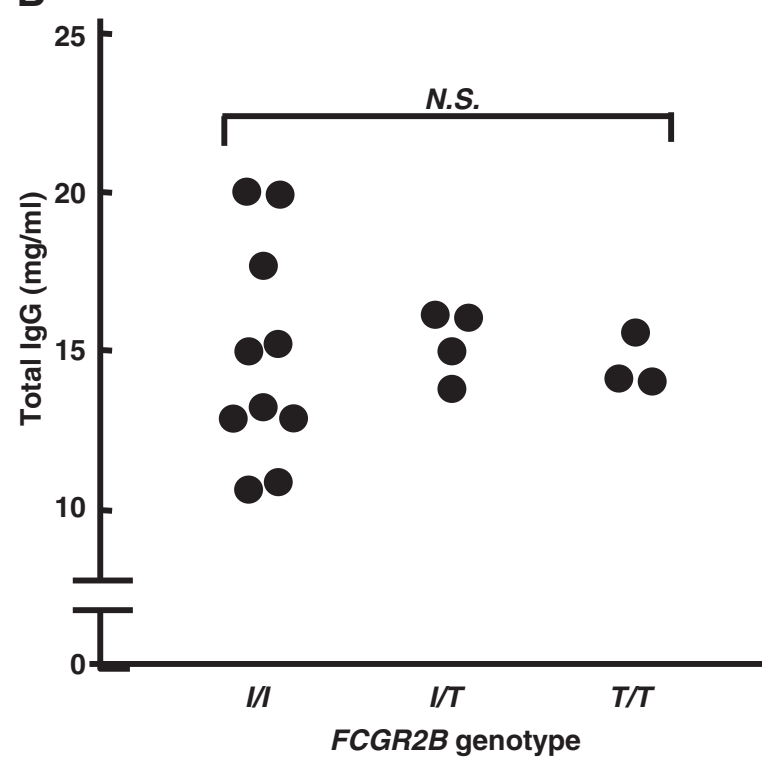

Figure 1 Serum IgG levels of healthy individuals. The concentrations of serum $\lg \mathrm{G}$ were measured by turbidimetric immunoassay. CD72 NS8-VNTR which was the tag polymorphism for human CD72 haplotypes was genotyped by PCR-simple sequence length polymorphism (SSLP) method, and FCGR2B-lle232Thr was genotyped by nested PCR and fluorescence resonance energy transfer (FRET) technology. The concentrations of serum lgG were compared among CD72 genotypes (A) or FCGR2B genotypes (B). The serum lgG levels were compared among individuals with $C D 72^{*} 1 / 1$, those with $C D 72^{*} 1 / 2$ and those with $C D 72 * 2 / 2$ by Kruskal-Wallis rank sum test, and P-value indicates the estimated probability of rejecting the null hypothesis that rank sum of the serum lgG levels among different CD72 haplotypes are not different; $P<0.05$. Additionally, concordance to the model in which the serum $\lg G$ levels are in proportion to the number of $C D 72^{*} 1$ haplotype was examined by regression analysis, and P-value indicates the estimated probability of rejecting the null hypothesis that serum lgG levels were not in proportion to the number of $C D 72^{*} 1$ haplotype; $P<0.01$. 
CD72 haplotypes by PCR-simple sequence length polymorphism (SSLP) method [7], and there were the total of 12 with $C D 72 * 1 / 1$, the total of 16 with $C D 72 * 1 / 2$, and the total of 2 with $C D 72 * 2 / 2$. The serum IgG level inversely correlated to the number of $C D 72 * 2$ allele with statistical significance (Figure 1A, regression analysis: $\mathrm{P}<0.01$; Kruskal-Wallis rank sum test: $\mathrm{P}<0.05)$. Additionally, we genotyped FCGR2B-Ile232Thr by nested PCR and fluorescence resonance energy transfer (FRET) technology in the same volunteers [12]. There was however no significant difference in the serum IgG level among FCGR2B-Ile232Thr genotypes (Figure 1B). Thus, CD72 polymorphism appears to play a role in the regulation of antibody production.

Human $\mathrm{CD} 72 \mathrm{fl}$ but not $\mathrm{CD} 72 \Delta \mathrm{ex} 8$ regulates $\mathrm{BCR}$ signaling To assess the signaling function of both human CD72fl and the CD72 $\mathrm{ex} 8$ isoforms, we constructed retroviral vectors containing FLAG-tagged CD72fl, CD72 $\Delta$ ex8 isoform, or their YF mutants in which the tyrosine residue at ITIM was replaced by phenylalanine. Although BCR ligation by antigen but not anti-Ig antibody induces efficient mouse CD72-mediated BCR regulation through recruitment of SHP-1, human B cell lines including Raji and Ramos exhibited reduced SHP-1 expression [13], and expressed endogenous CD72 and BCR with unknown antigen specificity [14]. We therefore transduced these retroviral vectors into the mouse B cell line K46 $\mathrm{v}$, which we used to examine the signaling function of mouse CD72 because of its expression of BCR reactive to the hapten (4-hydroxy-3-nitrophenyl) acetyl (NP) without endogenous CD72 expression [9]. All the K46 $\mathrm{V}$ transfectants expressed similar levels of NP-reactive $\mathrm{BCR}$ (Figure 2A), and BCR ligation by a specific antigen NP-BSA induced phosphorylation of extracellular signalregulated kinase (ERK) and $\mathrm{Ca}^{2+}$ influx (Figure 2B, C).

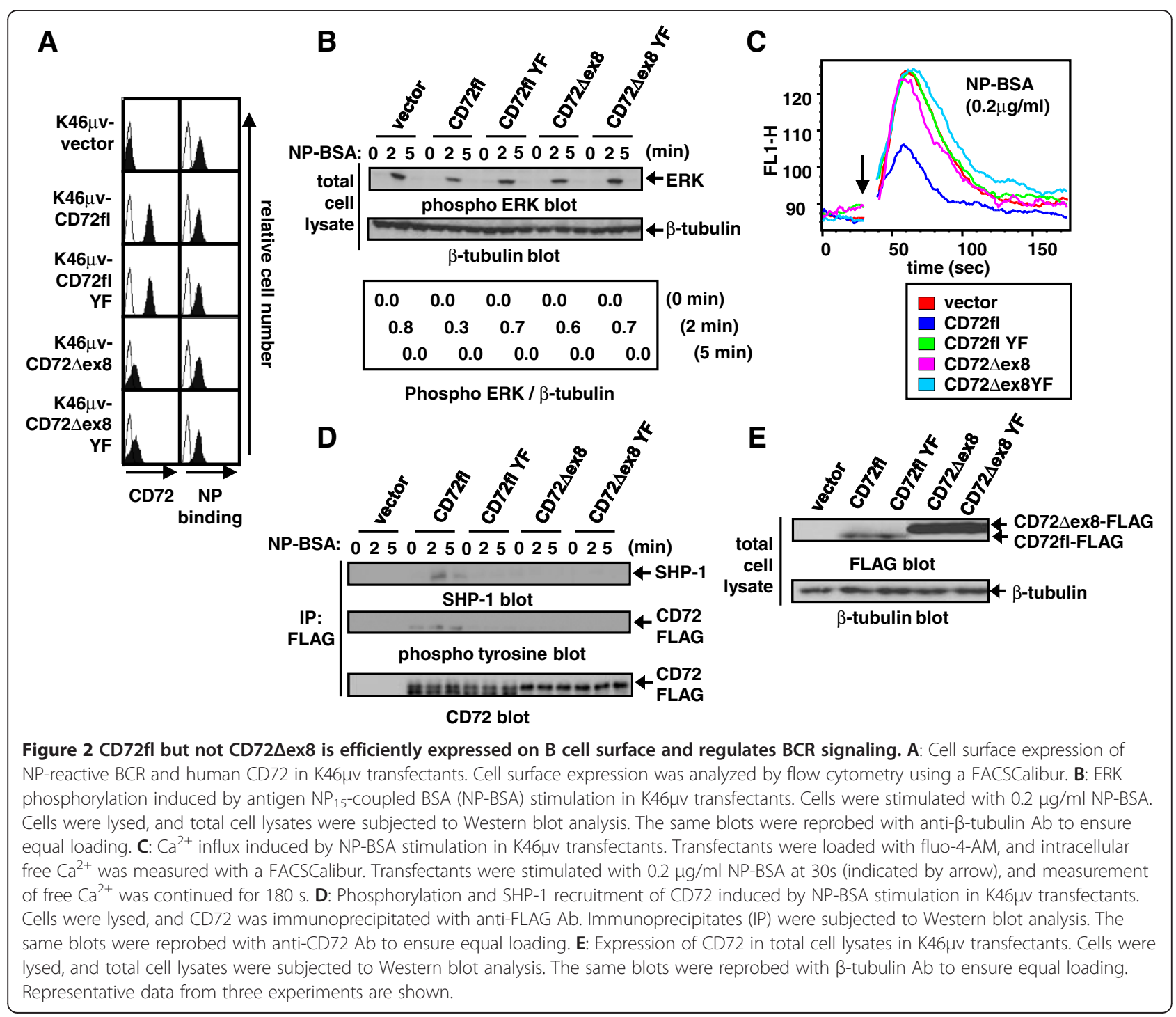


However, both ERK phosphorylation and $\mathrm{Ca}^{2+}$ influx induced by antigen stimulation were decreased in $\mathrm{K} 46 \mu \mathrm{v}$ $\mathrm{CD} 72 \mathrm{fl}$ transfectants compared to $\mathrm{K} 46 \mu \mathrm{v}$ cells trans-

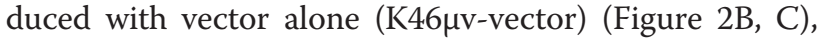
indicating that $\mathrm{CD} 72 \mathrm{fl}$ negatively regulates $\mathrm{BCR}$ signaling. In contrast, neither ERK phosphorylation nor $\mathrm{Ca}^{2+}$ flux was decreased in $\mathrm{K} 46 \mu \mathrm{v}$ transfectants that express the YF mutant of CD72fl (CD72flYF) (Figure 2B, C), indicating that $\mathrm{CD} 72 \mathrm{fl}$-induced $\mathrm{BCR}$ regulation depends on its ITIM. Accordingly, CD72fl but not CD72flYF was markedly phosphorylated and associated with SHP-1 after antigen stimulation (Figure 2D). Human CD72fl thus negatively regulates $B C R$ signaling in mouse $B$ cells by recruiting SHP-1 at the phosphorylated ITIM, as is the case for mouse CD72 [4-6].

In $\mathrm{K} 46 \mu \mathrm{v}$ transfectants, surface expression of $\mathrm{CD} 72 \Delta \mathrm{ex} 8$ isoform was much lower than that of CD72fl (Figure 2A), although the amount of CD72 $\Delta \operatorname{ex} 8$ in total cell lysates was much larger than that of CD72fl (Figure 2E), suggesting poor efficiency of transport of CD72 $\Delta$ ex8 to the cell surface. Equivalent ERK phosphorylation and $\mathrm{Ca}^{2+}$ influx by NP-BSA treatment in K46 $\mu \mathrm{v}$ CD72 $\triangle$ ex8 cells indicate that CD72 $\Delta$ ex8 does not regulate BCR signaling. $C D 72 \Delta \mathrm{ex} 8$ is thus poorly expressed on the cell surface and does not regulate BCR signaling.

\section{Increased $C D 72 \Delta$ ex8 expression in $C D 72 * 2$-carrying peripheral B-cells}

Since the ratio of CD72 $\triangle \mathrm{ex} 8 \mathrm{mRNA}$ to CD72fl mRNA is increased in $\mathrm{B}$ cells from $C D 72 * 2$-carrying individuals resistant to SLE [7], we next determined the protein levels of endogenous $\mathrm{CD} 72 \mathrm{fl}$ and $\mathrm{CD} 72 \Delta \mathrm{ex} 8$ in human primary B cells. When we examined the surface expression of CD72 by flow cytometry using an anti-CD72 antibody that reacts to both CD72fl and CD72 $\Delta$ ex8, peripheral blood B cells from healthy individuals carrying $C D 72 * 1 / 1, C D 72 * 1 / 2$, or $C D 72 * 2 / 2$ exhibited similar levels of surface CD72 expression (Figure 3A). This finding suggests that expression of CD72fl does not differ among various $C D 72$ haplotypes because CD72 $\Delta$ ex8 is only poorly expressed on the surface. To examine the CD72 $\Delta$ ex8 level, we generated polyclonal antibodies that recognize $\mathrm{CD} 72 \Delta \mathrm{ex} 8$ but not $\mathrm{CD} 72 \mathrm{fl}$, and measured the protein levels of $C D 72 \Delta$ ex 8 corrected for the level of $\beta$-tubulin. The CD72 $\Delta$ ex8 levels in peripheral blood B cells carrying $C D 72 * 2$ including both $C D 72 * 1 / 2$ and $C D 72 * 2 / 2$ were significantly higher than those carrying $C D 72 * 1 / 1$ (Figure 3B, C; $C D 72 * 1 / 1$ vs. $C D 72 * 1 / 2+* 2 / 2$, Mann-Whitney $U$ test: $P<0.05)$. Taken together, these findings indicate that $\mathrm{B}$ cells carrying $C D 72 * 2$, a haplotype protective against SLE, express higher levels of CD72 $\Delta$ ex8 whereas levels of CD72fl were similar regardless of the number of $C D 72 \% 2$. Increase in the CD72 $\triangle \mathrm{ex} 8$ level is thus responsible for $C D 72 * 2$-mediated resistance to SLE, although CD72 $\triangle$ ex8 does not regulate BCR signaling.

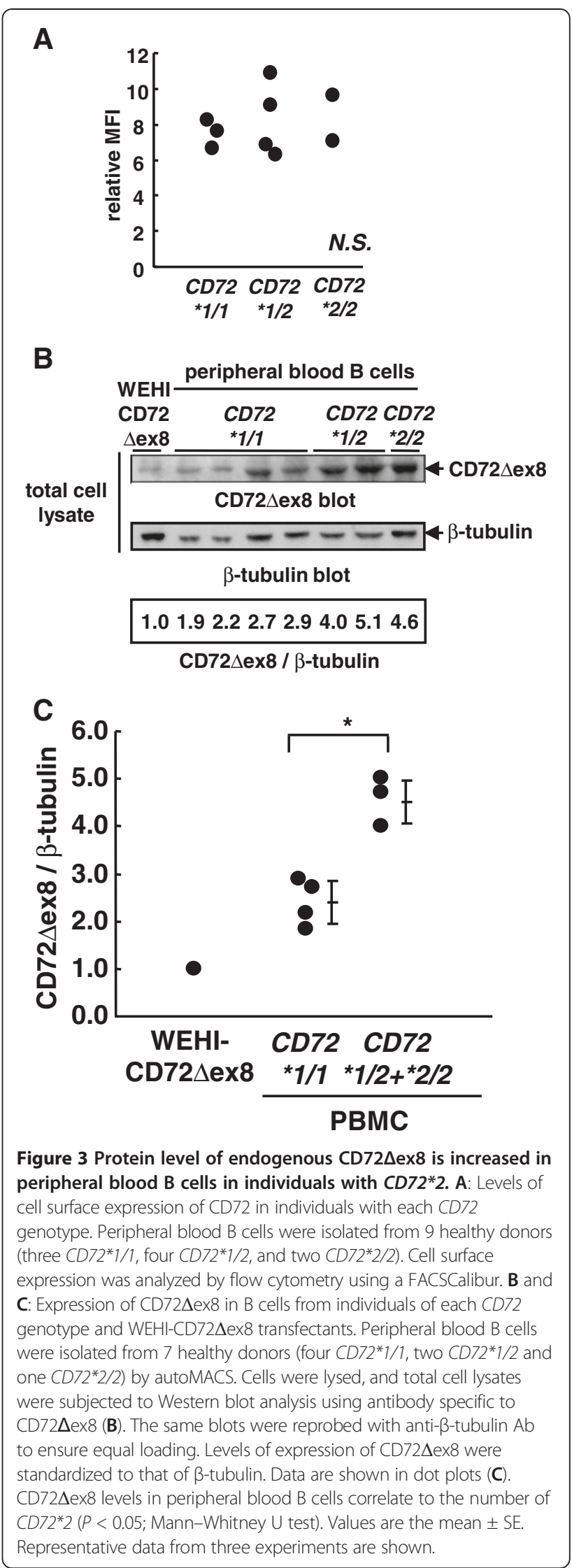




\section{CD72 $\Delta$ ex8 accumulates in ER}

Since CD72 $\mathrm{e}$ ex 8 is poorly expressed on the cell surface, we next examined the intracellular localization of CD72fl and CD72 $\Delta$ ex 8 by confocal microscopy. In mouse B-cell line WEHI-231 transfectants, CD72 $\Delta$ ex8 was accumulated inside the cells, though its location was unclear due to the relatively small cytoplasm (Figure 4). To determine intracellular localization in detail, we then transduced retroviral vectors encoding $\mathrm{CD} 72 \mathrm{fl}$ and $\mathrm{CD} 72 \Delta \mathrm{ex} 8$ into Balb/c-3T3 cells, which contain much larger cytoplasm than B lymphocytes. Fluorescence for CD72 was much stronger in CD72 2 ex8 transfectants than in CD72fl transfectants, indicating that CD72 $\mathrm{e}$ ex 8 is accumulated inside the cells (Figure 5A). We therefore amplified the fluorescence intensity of CD72fl to the levels of CD72 $\triangle \mathrm{ex} 8$, and compared the intracellular localization of these proteins. Both CD72fl and CD72 $\Delta$ ex8 were localized in ER, mitochondria, Golgi apparatus, early endosomes, and late endosomes, which are reasonable locations for membrane proteins (Figure $5 \mathrm{~B}$ ). However, CD72 $\mathrm{ex} 8$ was strongly accumulated in ER compared to CD72fl (Figure 5B).

Eukaryotic cells react rapidly to dysfunction of the ER through a set of evolutionarily conserved adaptive pathways known as the unfolded protein response (UPR), and persistent or intense ER stress triggers apoptosis $[15,16]$. To obtain additional evidence for ER localization of $C D 72 \Delta \mathrm{ex} 8$, we addressed whether CD72 $\Delta \mathrm{ex} 8$ augments ER stress-mediated apoptosis. Expression of FLAG-tagged CD72 $\mathrm{ex} 8$ but not CD72fl enhanced apoptosis of mouse B cell line WEHI-231 and human B cell line Raji induced by thapsigargin, which generates ER stress by depletion of $\mathrm{Ca}^{2+}$ from ER [17] (Figure 6A, B). In contrast, CD72 $\Delta \mathrm{ex} 8 \mathrm{did}$ not augment apoptosis of WEHI-231 transfectants induced by treatment with staurosporine, which induces apoptosis by mitochondrial stress [18] (Figure 6C). Thus, CD72 $\Delta$ ex8 specifically enhances ER stress-induced apoptosis.

\section{Discussion}

Here we addressed humoral immunity and expression of the two isoforms of human CD72, i.e., CD72fl and CD72 $\triangle \mathrm{ex} 8$, in healthy individuals carrying different $C D 72$ haplotypes as the coding sequences of these isoforms were the same regardless of different $C D 72$ haplotypes. Individuals carrying higher numbers of $C D 72 * 2$ allele showed lower serum levels of IgG, clearly demonstrating association of, $C D 72 * 2$ with low serum Ig as well as reduced risk for SLE [7]. The cell surface expression level of CD72fl on peripheral B cells was similar regardless of the number of $C D 72 * 2$ allele. This is in agreement with the previous study in which flow cytometry demonstrated relatively homogeneous CD72 expression on B cell surface in healthy individuals [19]. Thus, BCR signal regulation mediated by $\mathrm{CD} 72 \mathrm{fl}$ in B cells does not differ among each $C D 72$ genotypes because BCR regulation appears to occur on the cell surface. In contrast, Individuals carrying higher numbers of $C D 72 * 2$ allele showed higher levels of CD72 $\triangle \mathrm{ex} 8$ in peripheral B cells. Although the sample size of this analysis is relatively small, this result is in agreement with our previous study

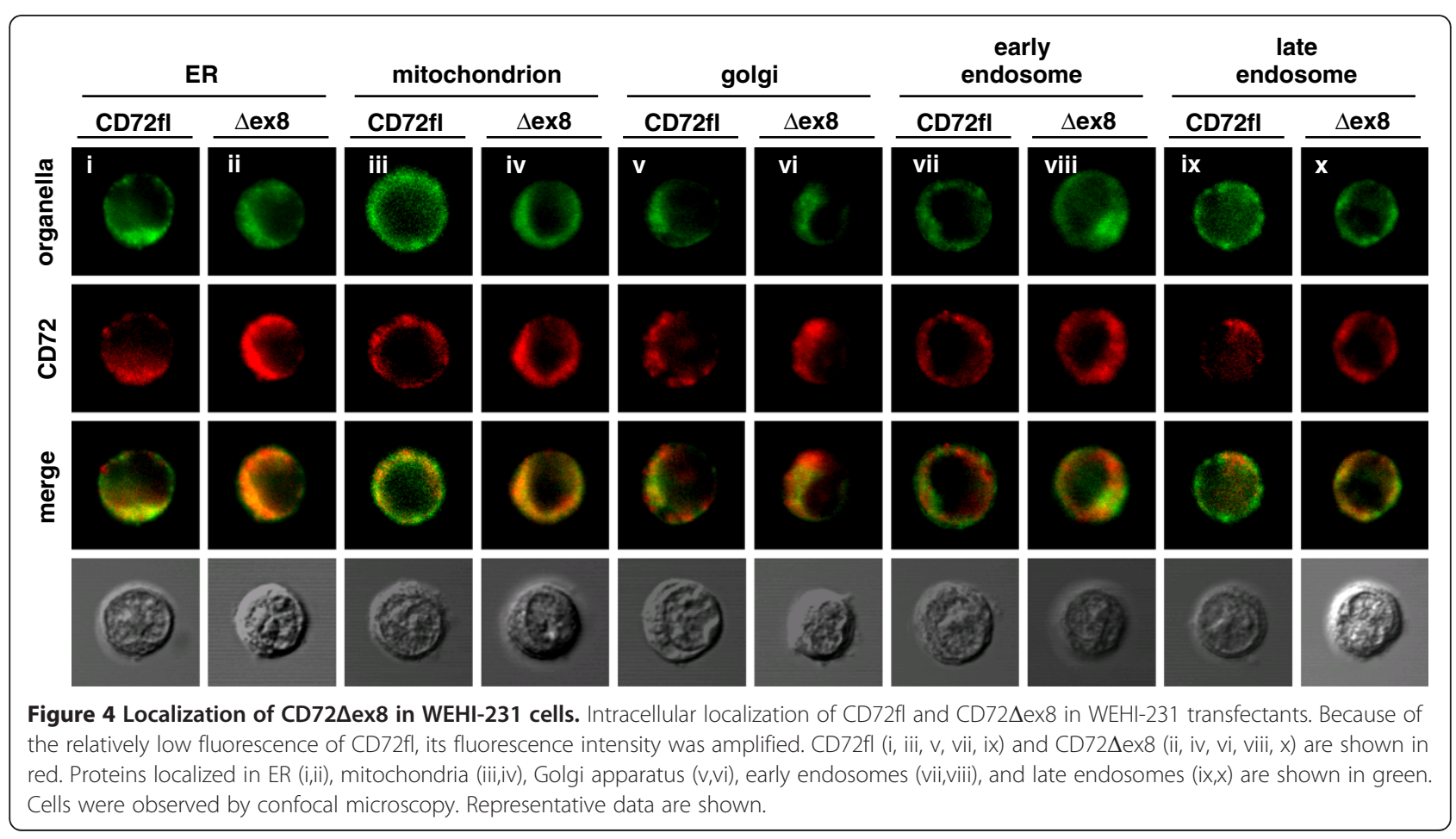




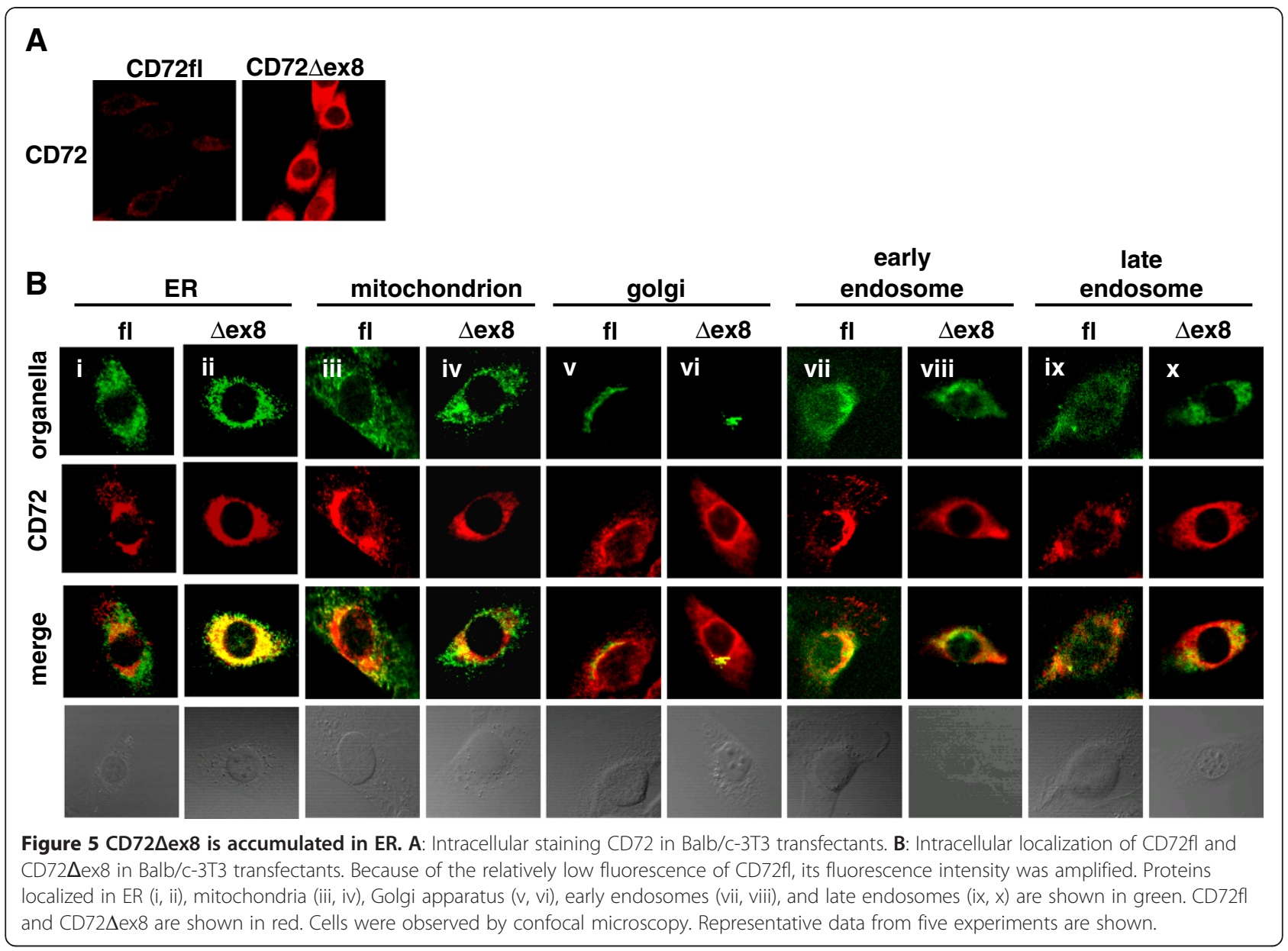

with a larger sample size $(n=32)$ demonstrating that the ratio of $C D 72 \Delta$ ex 8 to $C D 72 \mathrm{fl}$ mRNA correlates to the number of $C D 72 \% 2$ allele [7], and suggests that presence of $C D 72 * 2$ allele increases the protein level of CD72 $\Delta \mathrm{ex} 8$ but not CD72fl. Taken together, CD72 $\Delta$ ex8 but not CD72fl is responsible for difference in serum Ig and risk for SLE among individuals carrying different CD72 genotypes.

We further examined the functional activity of CD72fl and $C D 72 \Delta \mathrm{ex} 8$, in signal regulation and cellular localization by expressing these isoforms in mouse B cell lines K46 $\mathrm{v}$ and WEHI-231, human B cell line Raji and fibroblasts. As is the case for mouse CD72, CD72fl negatively regulated BCR signaling in ITIM-dependent fashion. In contrast, $\mathrm{CD} 72 \Delta \mathrm{ex} 8$ did not regulate $\mathrm{BCR}$ signaling, probably due to its poor surface expression. CD72 2 ex8 was accumulated in ER in fibroblasts and augmented apoptosis induced by ER-stress in WEHI-231 and Raji, demonstrating ER localization of CD72 $\Delta$ ex8. Thus, ER-localizing CD72 $\mathrm{ex} 8$ is responsible for the regulation of antibody production as well as susceptibility to SLE mediated by CD72 polymorphism.
CD72 4 ex 8 is generated by alternative splicing that skips exon 8, causing replacement of an exon 8-encoded stretch of 42 amino acids in the C-type lectin domain in the extracellular region by a totally different sequence of 49 amino acids encoded by exon 9 [7]. This extensive change in the extracellular $\mathrm{C}$-type lectin domain may cause accumulation of CD72 $\Delta$ ex 8 in ER. The change in the C-type lectin domain may induce misfolding of the CD72 $\Delta$ ex8 protein, which causes ER retention of the protein. Splicing isoforms of surface proteins such as human FcyRIb, mouse glutamate receptor 7b (GluR7b), and rat voltage-dependent and $\mathrm{Ca}_{2}^{+}$-activated $\mathrm{K}^{+}$channel (MaxiK) are mostly retained in the ER [20-22]. Whether these ER-localized isoforms play a role in cellular functions need to be elucidated in future studies.

We previously demonstrated that $C D 72 * 2$ specifically abrogates the susceptibility to SLE conferred by the allele of FcyRIIb carrying the substitution of 232Ile by 232Thr (FCGR2B-Ile232Thr) [7], which is suggested to reduce the inhibitory function of Fc $\gamma$ RIIb [23]. FcyRIIbdeficient mice exhibit loss of $B$ cell self-tolerance at the $\mathrm{IgG}^{+} \mathrm{B}$ cell and plasma cell stages, and development of 


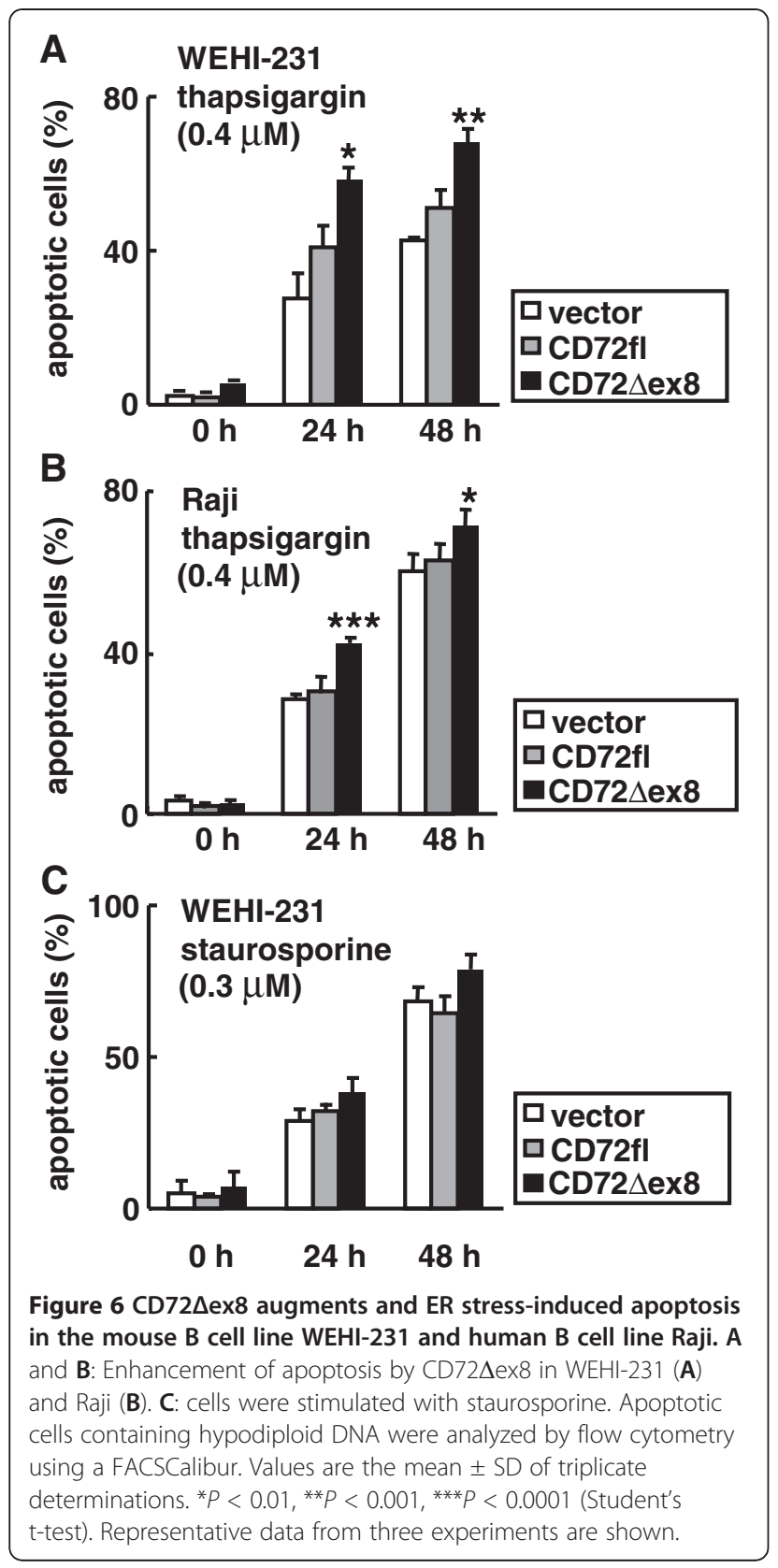

SLE-like disease [24], demonstrating that Fc $\gamma$ RIIb provides a distal peripheral checkpoint limiting the accumulation of self-reactive plasma cells and thereby preventing SLE. The Ile232Thr substitution may thus reduce the activity of FcyRIIb in maintaining self-tolerance, and thereby increase risk for SLE. Our findings suggest that $C D 72 \% 2$ augments apoptosis of $\mathrm{B}$ cells by enhancing expression of CD72 $\triangle$ ex8 (Figure 6). Augmented $\mathrm{B}$ cell apoptosis may reduce the number of selfreactive $\mathrm{B}$ cells, and autoantibody-producing plasma cells may not be generated at high frequency in CD72 2 carrying individuals even in the presence of inefficient self-tolerance caused by FCGR2B-Ile232Thr. Recently, over 30 loci were reported as the genes significantly associated with susceptibility to SLE by genome-wide association study (GWAS) [25]. However, most of these loci carry very small effect, and the proportion of heritability explained by these variants is modest [26,27]. Other than the effect of causal rare variant, it is possible to explain a part of "missing heritability" by the genegene interaction. This study, which shows the functional interaction between $C D 72$ and $F C G R 2 B$, will be a functional evidence of such a gene-gene interaction.

Increasing numbers of studies have identified diseasesusceptibility alleles associated with specific splicing patterns [28]. Our previous [7] and the present studies have demonstrated that the genetic polymorphism of human CD72 associated with susceptibility to SLE regulates the efficiency of alternative splicing, although how the polymorphism affects alternative splicing is not known. Further elucidation of the molecular mechanisms of splice variations and their functional significance will provide important clues to the pathogenesis of autoimmune diseases and development of new therapeutic approaches.

\section{Conclusion}

Our finding that serum IgG levels in individuals carrying the $C D 72 * 2$ allele including both individuals with $C D 72 * 1 / 2$ and those with $C D 72 * 2 / 2$ are significantly lower than those in individuals carrying $C D 72 * 1 / 1$ clearly demonstrates that human $C D 72$ polymorphism regulates humoral immunity as well as risk for SLE [7]. Of the two splicing isoforms of human CD72, CD72 $\Delta$ ex8 appears to be responsible for the regulation of Ig production and autoimmunity, as the expression level of CD72 $\Delta$ ex8 but not the other isoform CD72fl is different between B cells from individuals with $C D 72 * 2$ and those with $C D 72 * 1 / 1$. Because CD72 $\triangle$ ex 8 is accumulated in ER and poorly transported to the cell surface, $C D 72 \Delta$ ex 8 may regulate $B$ cell activity by a mechanism distinct from CD72fl that is expressed on the surface and regulate signaling through $\mathrm{BCR}$ as is the case for mouse CD72. Although overexpression of CD72 $\triangle$ ex8 enhances ER stress-induced apoptosis, further studies are required to elucidate how $\mathrm{CD} 72 \Delta \mathrm{ex} 8$ in ER regulates B cell activity.

\section{Abbreviations \\ BCR: B cell antigen receptor; ER: Endoplasmic reticulum; ERK: Extracellular signal-regulated kinase; GWAS: Genome-wide association study: \\ Ig: Immunoglobulin; ITIM: Immunoreceptor tyrosine-based inhibition motif; NP: Hapten (4-hydroxy-3-nitrophenyl) acetyl; SHP-1: Src homology 2 domain- containing protein tyrosine phosphatase-1; SLE: Systemic lupus erythematosus.}

\section{Competing interest}

The authors declare that they have no competing interests.

\section{Authors' contributions}

Study design: YH, TA, NT, ZH, KT, $\Pi$; data analysis: YH, TA, TT; manuscript writing: YH, NT, KT, TT; patient recruitment: NT; collecting data: YH, TA, NT. All authors read and approved the final manuscript. 


\section{Acknowledgement}

We thank Dr. T. Kitamura (The University of Tokyo, Japan) for providing Plat-E and Plat-A cells, Drs. M. Hagiwara, S. Shimizu, T. Mizuta (Tokyo Medical and Dental University, Japan), T. Suzuki (Mitsui Memorial Hospital, Japan), D. Sakurai (Dokkyo Medical University, Japan) and A. Fujimoto (RIKEN, Japan) for valuable suggestions, and K. Mizuno and A. Yoshino (Tokyo Medical and Dental University, Japan) for technical assistance.

This work was supported in part by grants from the Ministry of Education, Culture, Sports, Science, and Technology of Japan and the Japan Society for the Promotion of Science.

\section{Author details}

'Laboratory of Immunology, School of Biomedical Science, Tokyo Medical and Dental University, 1-5-45 Yushima, Bunkyo-ku, Tokyo 113-8510, Japan. ${ }^{2}$ Department of Immunology, Medical Research Institute, Tokyo Medical and Dental University, 1-5-45, Yushima, Bunkyo-ku, Tokyo 113-8510, Japan. ${ }^{3}$ Department of Human Genetics, Graduate School of Medicine, The University of Tokyo, 7-3-1, Hongo, Bunkyo-ku, Tokyo 113-0033, Japan. ${ }^{4}$ Department of Allergy and Rheumatology, Graduate School of Medicine, The University of Tokyo, 7-3-1, Hongo, Bunkyo-ku, Tokyo 113-0033, Japan. ${ }^{5}$ Doctoral Program in Life System Medical Sciences, Graduate School of Comprehensive Human Sciences, University of Tsukuba, 1-1-1, Tennodai, Tsukuba 305-8575, Japan. ${ }^{6}$ Core Research for Evolutional Science and Technology, Japan Science and Technology Agency, 4-1-8, Honcho, Kawaguchi 332-0012, Japan.

Received: 17 September 2012 Accepted: 21 December 2012 Published: 26 December 2012

\section{References}

1. Nakayama E, von Hoegen I, Parnes JR: Sequence of the Lyb-2 B-cell differentiation antigen defines a gene superfamily of receptors with inverted membrane orientation. Proc Natl Acad Sci USA 1989, 86:1352-1356.

2. Von Hoegen I, Nakayama E, Parnes JR: Identification of a human protein homologous to the mouse Lyb-2 B cell differentiation antigen and sequence of the corresponding CDNA. J Immunol 1990, 144:4870-4877.

3. Beavil AJ, Edmeades RL, Gould HJ, Sutton BJ: a-Helical coiled-coil stalks in the low-affinity receptor for $\lg \mathrm{E}(\mathrm{FcERII} / \mathrm{CD} 23)$ and related C-type lectins. Proc Natl Acad Sci USA 1992, 89:753-757.

4. Adachi T, Flaswinkel H, Yakura H, Reth M, Tsubata T: The B cell surface protein $\mathrm{CD} 72$ recruits the tyrosine phosphatase SHP-1 upon tyrosine phosphorylation. J Immunol 1998, 160:4662-4665.

5. Adachi T, Wakabayashi C, Nakayama T, Yakura H, Tsubata T: CD72 negatively regulates signaling through the antigen receptor of $B$ cells. J Immunol 2000, 164:1223-1229.

6. Adachi T, Wienands J, Wakabayashi C, Yakura H, Reth M, Tsubata T: SHP-1 requires inhibitory co-receptors to down-modulate $B$ cell antigen receptor-mediated phosphorylation of cellular substrates. J Biol Chem 2001, 276:26648-26655.

7. Hitomi Y, Tsuchiya N, Kawasaki A, Ohashi J, Suzuki T, Kyogoku C, Fukazawa T, Bejrachandra S, Siriboonrit U, Chandanayingyong D, et al: CD72 polymorphisms associated with alternative splicing modify susceptibility to human systemic lupus erythematosus through epistatic interaction with FCGR2B. Hum Mol Genet 2004, 13:2907-2917.

8. Tsuchiya N, Honda Z, Tokunaga K: Role of B cell inhibitory receptor polymorphisms for systemic lupus erythematosus: a negative times a negative makes a positive. J Hum Genet 2006, 51:741-750.

9. Wakabayashi C, Adachi T, Wienands J, Tsubata T: A distinct signaling pathway used by the lgG-containing B cell antigen receptor. Science 2002, 298:2392-2395.

10. Yan BC, Adachi T, Tsubata T: ER stress is involved in B cell antigen receptor ligation-induced apoptosis. Biochem Biophys Res Commun 2008, 365:143-148.

11. Tokunaga K, Imanishi T, Takahashi K, Juji T: On the origin and dispersal of East Asian populations as viewed from HLA haplotypes. In Prehistoric Mongoloid Dispersals. Edited by Akazawa T, Szathmary EJ. Oxford: Oxford University Press; 1996:187-197.

12. Kyogoku C, Dijstelbloem HM, Tsuchiya N, Hatta Y, Kato H, Yamaguchi A, Fukazawa T, Jansen MD, Hashimoto H, van de Winkel JG, Kallenberg CG, Tokunaga K: Fcgamma receptor gene polymorphisms in Japanese patients with systemic lupus erythematosus: contribution of FCGR2B to genetic susceptibility. Arthritis Rheum 2002, 46:1242-1254.
13. Delibrias CC, Floettmann JE, Rowe M, Dearon DT: Downregulated expression of SHP-1 in Burkitt lymphomas and germinal center B lymphocytes. J Exp Med 1997, 186:1575-1583.

14. Schwarting R, Castello R, Moldenhauer G, Pezzutto A, von Hoegen I, Ludwig WD, Parnes JR, Dörken B: Human Lyb-2 homolog CD72 is a marker for progenitor B-cell leukemias. Am J Hematol 1992, 41:151-158.

15. Schröder M, Kaufman RJ: The unfolded protein response in nutrient sensing and differentiation. Annu Rev Biochem 2005, 74:739-789.

16. Ron D, Walter P: Signal integration in the endoplasmic reticulum unfolded protein response. Nat Rev Mol Cell Biol 2007, 8:519-529.

17. Wong WL, Brostrom MA, Kuznetsov G, Gmitter-Yellen D, Brostrom CO: Inhibition of protein synthesis and early protein processing by thapsigargin in cultured cells. Biochem J 1993, 289:71-79.

18. Bertrand R, Solary E, O'Connor P, Kohn KW, Pommier Y: Induction of a common pathway of apoptosis by staurosporine. Exp Cell Res 1994, 211:314-321.

19. Smith AJ, Gordon TP, Macardle PJ: Increased expression of the B-cellregulatory molecule CD72 in primary Sjögren's syndrome. Tissue Antigens 2004, 63:255-259.

20. van Vugt MJ, Reefman E, Zeelenberg I, Boonen G, Leusen JH, van de Winkel JG: The alternatively spliced CD64 transcript FcyRlb2 does not specify a surface-expressed isoform. Eur J Immunol 1999, 29:143-149.

21. Jaskolski F, Normand E, Mulle C, Coussen F: Differential trafficking of GluR7 Kainate receptor subunit splice variants. J Biol Chem 2005, 280:22968-22976.

22. Zarei MM, Zhu N, Alioua A, Eghbali M, Stefani E, Toro L: A novel MaxiK splice variant exhibits dominant-negative properties for surface expression. J Biol Chem 2001, 276:16232-16239.

23. Kono H, Kyogoku C, Suzuki T, Tsuchiya N, Honda H, Yamamoto K, Tokunaga K, Honda Z: FcyRllb lle232Thr transmembrane polymorphism associated with human systemic lupus erythematosus decreases affinity to lipid rafts and attenuates inhibitory effects on B cell receptor signaling. Hum Mol Genet 2005, 14:2881-2892.

24. Fukuyama H, Nimmerjahn F, Ravetch JV: The inhibitory Fcy receptor modulates autoimmunity by limiting the accumulation of immunoglobulin $\mathrm{G}^{+}$anti-DNA plasma cells. Nat Immunol 2005, 6:99-106.

25. Deng Y, Tsao BP: Genetic susceptibility to systemic lupus erythematosus in the genomic era. Nat Rev Rheumatol 2010, 6:683-692.

26. Goldstein DB: Common genetic variation and human traits. N Engl J Med 2009, 360:1696-1698.

27. Cirulli ET, Goldstein DB: Uncovering the roles of rare variants in common disease through whole-genome sequencing. Nat Rev Genet 2010, 11:415-425.

28. Wang GS, Cooper TA: Splicing in disease: disruption of the splicing code and the decoding machinery. Nat Rev Genet 2007, 8:749-761.

doi:10.1186/1471-2172-13-72

Cite this article as: Hitomi et al:: Human CD72 splicing isoform responsible for resistance to systemic lupus erythematosus regulates serum immunoglobulin level and is localized in endoplasmic reticulum. BMC Immunology 2012 13:72.

\section{Submit your next manuscript to BioMed Central and take full advantage of:}

- Convenient online submission

- Thorough peer review

- No space constraints or color figure charges

- Immediate publication on acceptance

- Inclusion in PubMed, CAS, Scopus and Google Scholar

- Research which is freely available for redistribution 